\title{
Design of Recycling System Based on Courier Package Recycling Equipment
}

\author{
Jiagui Xie ${ }^{1, *}$ Qin Chen ${ }^{2}$ Yu Yang ${ }^{3}$ \\ ${ }^{1}$ Department of Transportation, Southwest Jiaotong University Hope College, Jintang, Chengdu, Sichuan, China \\ ${ }^{2}$ Department of Transportation, Southwest Jiaotong University Hope College, Jintang, Chengdu, Sichuan, China \\ ${ }^{3}$ Department of Transportation, Southwest Jiaotong University Hope College, Jintang, Chengdu, Sichuan, China \\ *Corresponding author.Email: author@example.com
}

\begin{abstract}
Firstly, this paper gives the research background of this paper from two aspects: the two problems brought to the society by the development of e-commerce logistics and the shortcomings of the recovery research of express delivery packaging. Secondly, according to the investigation and data analysis, the problem of the current recovery system of express packaging is obtained, and the design idea of the recovery system of express packaging based recovery equipment is proposed. Finally, from the software and hardware basis, conceptual model, operating process, equipment use, operation mode, chain of interests, Expected effect, etc. The contents of this system are analyzed and elaborated completely.
\end{abstract}

Keywords: Courier package recycling, recycling equipment, recycling system

\section{BACKGROUND}

Since the beginning of the outbreak, the frequency of people going out shopping has been much reduced compared to previous years. The society has once again set off the upsurge of online shopping, online shopping has even become an essential part of the lives of many people. According to relevant data statistics, the number of express delivery purchases in China is continuously increasing at about 10 billion units per year. The growth of express delivery business will inevitably bring more and more use of parcel materials, which will bring two major problems to the society. Firstly, environmental pollution. According to relevant data, each express packaging box will produce $0.2 \mathrm{~kg}$ of solid waste, in the consumption of all express packaging, the general carton consumes nearly 10 million trees per year[1]. Secondly, the supply rate of packaging materials has been slower than the growth rate of express delivery business volume, significantly in short supply[2][3]. However, express packaging recycling is an effective way to solve the two problems facing the above society at the same time.

In recent years, there have been more and more studies on express packaging recycling, such as sustainable packaging, recycling and reuse, green express delivery, express packaging cycle economy, recyclable express box, recycling system and so on[4]. Among them, the analysis of the growth of the number of express delivery packages, packaging material recovery status quo, recovery difficulties and other content has been relatively comprehensive. At the same time, a small number of scholars have also proposed some recovery measures including policy incentives, intensifying publicity to improve industry consensus, green packaging technology innovation, improving environmental awareness, setting up express carton recovery points near the neighborhood, and establishing recovery enterprises[5][6]. However, these recovery measures have two disadvantages that are not conducive to implementation: On the one hand, there is a lack of systematic linkage, there is no main body of recovery measures that can take the lead in implementation, resulting in mutual evasion and thus making these recovery measures unable to be implemented. On the other hand, there is a lack of implementation and no existing reference system, resulting in that these measures are not implemented due to similar reasons such as unknown benefits and risks.

In summary, aiming at the above problems, this paper puts forward the idea of recovery system design based on express packaging recovery equipment on the basis of "Internet +" and Internet of Things technology, solves the above problems, and also further enriches the achievements in the field of express packaging recovery research. 


\section{INVESTIGATION AND ANALYSIS}

\subsection{Investigation Content}

In order to serve the design of the recovery system based on the express packaging recovery equipment, a questionnaire survey was first targeted (In April 2020, a set of questionnaires with the theme of "Return of Courier Package" was set up on this website of Questionnaire Star, and the website of the questionnaire was https://www.wjx.cn/vj/tj27XAY.aspx), the main contents of investigation and research are: the age distribution of online shopping groups, the number of online shopping in the last month, the attitude towards the recovery of express packaging, the treatment of express packaging after removing the package, whether the express packaging will be placed in the corresponding garbage box according to the category of garbage classification box, the willingness to use the recovery of express packaging and recovery equipment and the incentive mechanism, and the address selection for the establishment of express packaging recovery equipment.

\subsection{Analysis of Results of Investigations and Studies}

The specific data statistics of this investigation are shown in Table 1.
Some survey statistics from Table 1 show:

1) Online shopping groups are very younger. From the survey data of question 1 , it can be seen that more than $90 \%$ of the young people under 30 years of age, indicating that the recovery of express delivery packaging is mainly aimed at young people.

2 ) Consumption of the courier package is high. Question 2 survey data show that from the number of packages in the last month, the number of packages per 234 people is more than 2,000 , with an average of nearly 10 packages per person per month, that is, one package will be received every 3 days.

3 ) Courier package recycling attitude is better than action. The survey data of Questions 3-5 showed that $76 \%$ of the people expressed their active support for the recycling of express packaging, of which only about $12 \%$ were taking action. After unpacking, most people will directly throw the express package into the garbage can or hand it to a specially-assigned person. Fortunately, more than $80 \%$ of people are willing to deliver it according to the garbage classification. However, due to the serious formalization of garbage classification, they do not carry out classified recycling according to the garbage classification. Moreover, only a part of the express package is picked up garbage people sell to the waste recycling station for recycling, the existing express package recycling system is shown in Figure 1.

Table 1. Questionnaire Data Summary for "Courier Package Return" Topic

\begin{tabular}{|l|l|}
\hline Questionnaire Title & Questionnaire Data Statistics \\
\hline Question 1 Your Age? & $\begin{array}{l}\text { Of those who participated in the questionnaire, about } \\
91.9 \% \text { were young people under 30 years of age. }\end{array}$ \\
\hline $\begin{array}{l}\text { Question } 2 \text { In the past month, the frequency of online } \\
\text { shopping? }\end{array}$ & $\begin{array}{l}\text { According to statistics, the total number of express } \\
\text { packages in the past month for 234 questionnaire } \\
\text { respondents was 2069. }\end{array}$ \\
\hline Question 3 Attitude to courier package recycling? & $\begin{array}{l}\text { About 76\% indicated active support for the recovery of the } \\
\text { courier package. }\end{array}$ \\
\hline $\begin{array}{l}\text { Question } 4 \text { How to handle the express package after } \\
\text { unpacking? }\end{array}$ & $\begin{array}{l}\text { About 90\% of people put the express package into the } \\
\text { garbage can, to a specially-assigned person to recycle, } \\
\text { everywhere to throw. }\end{array}$ \\
\hline $\begin{array}{l}\text { Question } 5 \text { Will the courier packages be placed in the } \\
\text { appropriate trash bin by category based on the trash } \\
\text { bin? }\end{array}$ & $\begin{array}{l}\text { About 80\% of people are ideologically willing to dispose of } \\
\text { express packages according to garbage classification. }\end{array}$ \\
\hline $\begin{array}{l}\text { Question } 6 \text { If a courier pack recovery device is set up, } \\
\text { would it be willing to use it? }\end{array}$ & $\begin{array}{l}\text { More than 90\% of people are willing to put the courier } \\
\text { package into the courier parcel processing equipment. }\end{array}$ \\
\hline $\begin{array}{l}\text { Question } 7 \text { Which of the following methods motivates } \\
\text { you to use recycling equipment? }\end{array}$ & $\begin{array}{l}\text { About one-third chose to return cash red envelopes, and } \\
\text { about one-third chose to return Integral on the platform. }\end{array}$ \\
\hline $\begin{array}{l}\text { Question } 8 \text { Would you prefer a courier package } \\
\text { recycling device to be located next to the trash can? }\end{array}$ & $\begin{array}{l}\text { About 95\% prefer recycling equipment to be located next to } \\
\text { garbage bins. }\end{array}$ \\
\hline
\end{tabular}




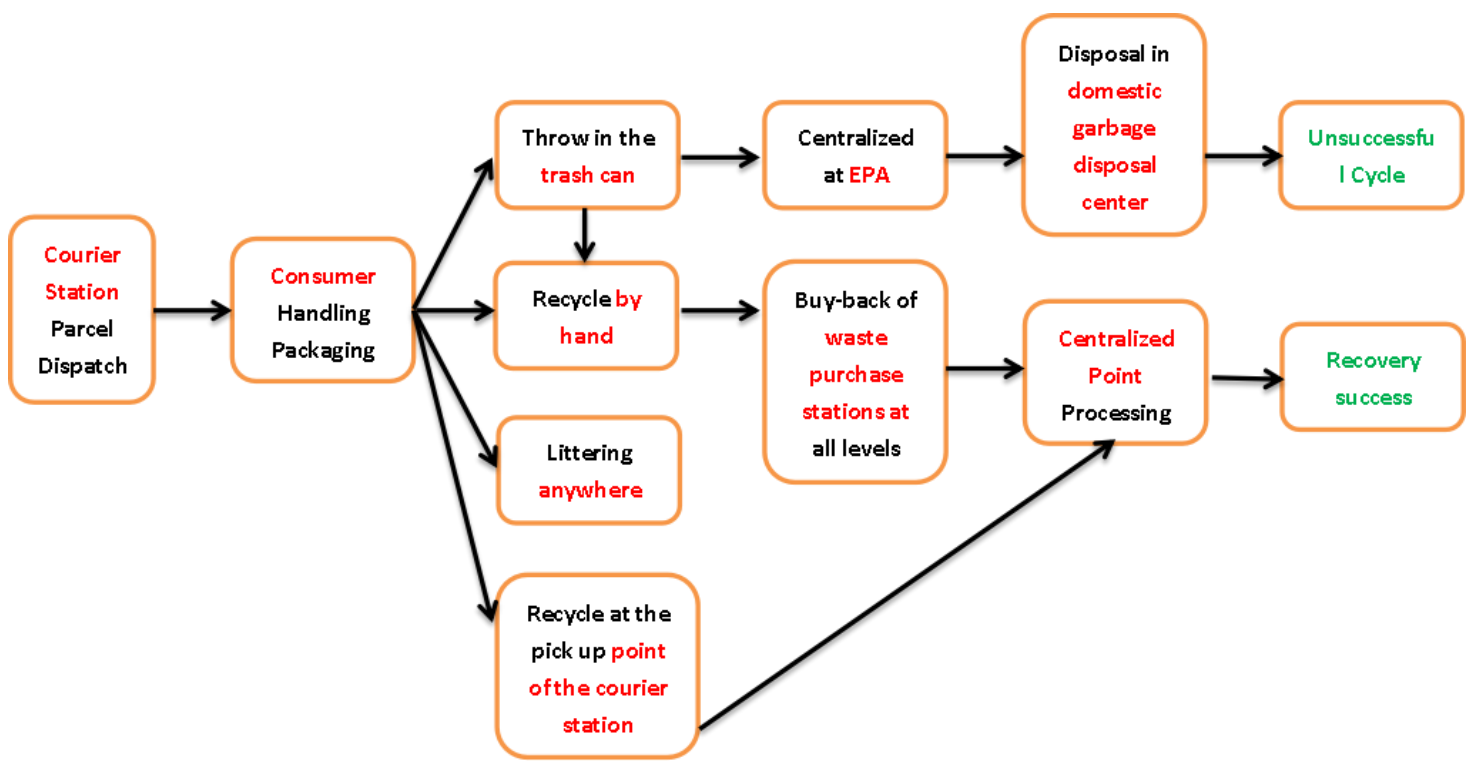

Figure 1 Existing courier packaging recycling system

As shown in Figure 1, although the existing express packaging recycling system has a network structure, in this network, each express packaging recycling chain can basically exist independently, with few intersections and connections, which is similar to "fragmented" and is not conducive to resource integration and optimal allocation, resulting in waste of resources and reducing profit margin.

4 ) The express package cannot be completely separated from the garbage, and effective recycling is difficult. The survey results found that about $90 \%$ of people choose to throw the express package into the garbage can, give it to a specially-assigned person for recycling, and throw it anywhere. Obviously, there are many and non-professional treatment channels, which cannot be completely diverted from garbage, resulting in difficulty in effective recovery.

5 ) Courier packaging recovery equipment is beneficial to improve recovery. As the survey data in question 6 shows, more than $90 \%$ of people are willing to put express packaging into the device. Compared with the existing recovery system, the use of recovery equipment for recovery, centralized channels and high degree of specialization, can achieve the diversion of express packaging and garbage, can well guide people to recover express packaging, and can significantly improve the effective recovery rate of express packaging.

6) In order to facilitate express packaging recycling, through the survey found that about $95 \%$ of people prefer recycling equipment set next to the garbage disposal bucket. At the same time, in order to encourage more people to actively recycle express packaging, the investigation is specially carried out for the incentive mode. It is found that about $1 / 3$ of the people choose the way of returning cash red envelopes, about $1 / 3$ of the people choose various points of the return platform, and of course, about $1 / 3$ of the people are willing to deliver to the recycling equipment to support the recycling of express packaging even if there is no reward.

\subsection{Innovative Thinking}

In summary, the existing express packaging recovery system has the shortcomings of scattered and nonprofessional recovery mode, not conducive to the recovery data statistics and the optimal allocation of resources, low effective recovery (the effective recovery in this paper refers to the recovery that can be directly used), no incentive, etc. At the same time, the preliminary investigation on the establishment of express packaging recovery equipment proposed in this paper is in good condition, and everyone has given a positive response to the willingness to recover, the location of the establishment of recovery equipment, and the incentive way to encourage recovery. In this way, the idea of establishing express packaging recovery equipment is of research significance.

The basic problem-solving idea of this paper is shown in Figure 2.

\section{CONSTRUCTION OF RECOVERY SYSTEM BASED ON COURIER PACKAGE RECOVERY EQUIPMENT}

\subsection{Software and Hardware Basis of the System}

The recovery system is mainly composed of express packaging recovery equipment, relevant Internet of Things technical equipment and other hardware and "Internet + " technical software. 


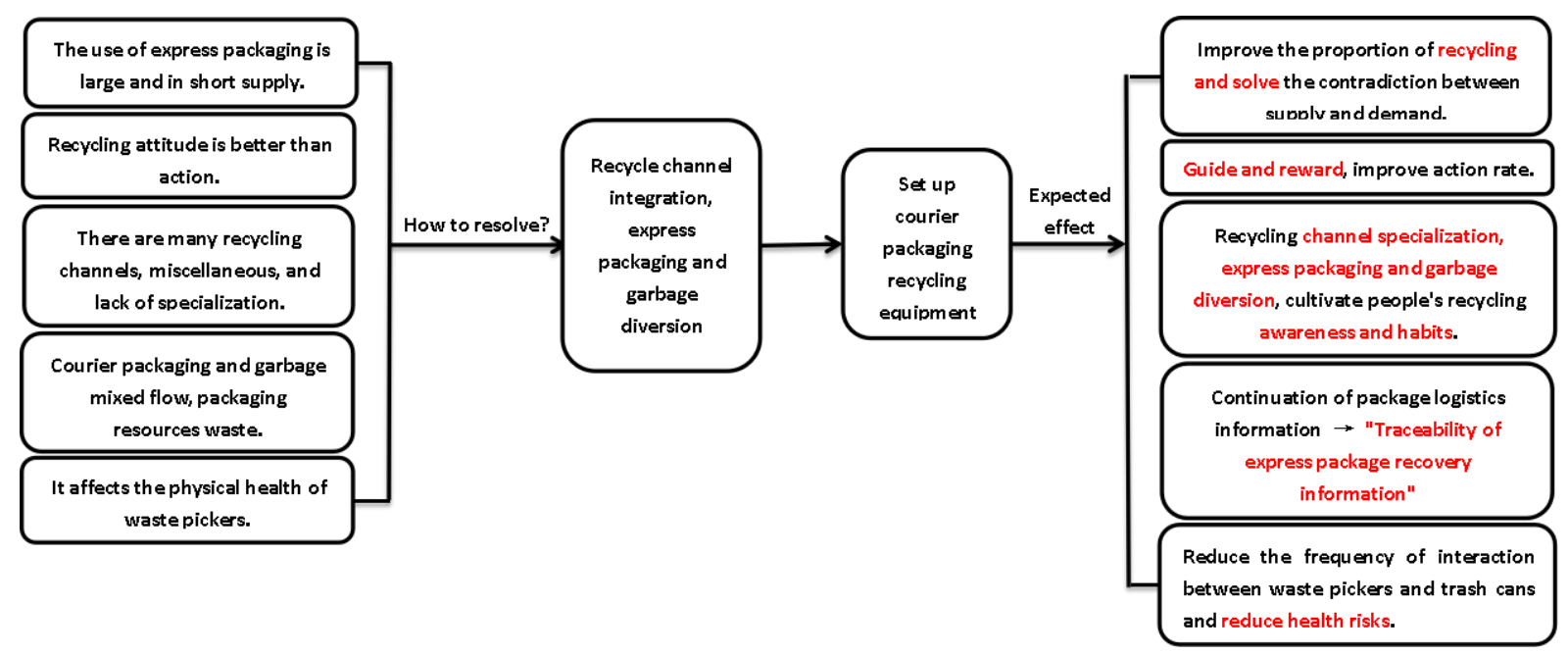

Figure 2 Innovative thinking on the design of express packaging recycling system

\section{1) Courier Package Recovery Equipment}

Courier packaging recycling equipment is the main body of recycling system. In the era of scientific and technological innovation, the research related to express packaging recovery equipment has achieved results. Based on the search for a series of keywords such as "express packaging recovery equipment", about 40 relevant patents have been found through the patent star retrieval system query of China Patent Information Center, including single recovery, incidental recovery of other functions, involving code sweeping, app or cloud detection, etc. These results have been relatively mature and can be selectively improved and optimized and applied to this system.

\section{2) Internet of Things Technology}

Internet of Things technology is an essential auxiliary technology in recycling systems. In the recycling system, we need to realize the functions of express packaging identification, storage, information transmission, positioning, etc. through the Internet of Things technology. At present, the Internet of Things technology for these functions has been very mature, such as intelligent carton recycler (patent number ZL201920873191.3), an intelligent recycling cabinet for express packaging recycling (patent number CN201820940528.0), which have integrated the Internet of Things technology.

\section{3 ) "Internet +" Technology}

"Internet $+"$ technology is an essential support technology in the express packaging recovery system. In this recycling system, we need the relevant management information system or platform, and then use the mobile phone APP client to realize the information sharing and interaction among the recovery device, APP and management information platform through the "Internet $+"$ technology. At present, these technologies have been relatively mature in the use of sharing bicycles, Gaode maps, Didi taxi, etc., and it is easy to apply them to our recovery system.

\subsection{Conceptual Model of the System}

According to our design idea, "recycling channel integration, express packaging and garbage diversion" as the core idea to solve the traditional recycling system problems. The recovery model of the recovery device based on the courier package is shown in Figure 3.

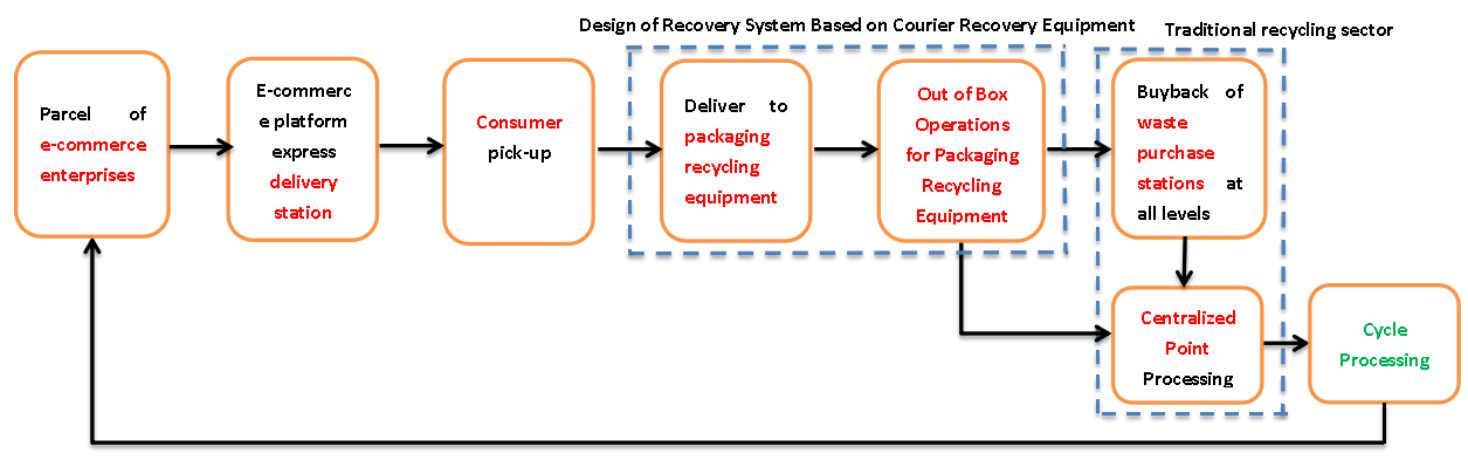

Figure 3 System Based on Courier Package Recycling Equipment 


\subsection{Operating Process of System}

\section{1) Parcel of E-commerce Enterprises}

E-commerce enterprises are the source of express delivery, here, online shopping consumers from Tmall, JD, VIPS and other e-commerce platforms to buy commodities. Enterprises purchase packaging from the suppliers of relevant packaging materials (including packaging supply after cycle treatment), flow the commodities downstream in the form of express delivery package, and reach the express stations (express delivery points) of "the last kilometer".

\section{2) Express Sites of Each E-commerce Platform}

Each express delivery station shall distribute express delivery according to the information of customer receipt. At this time, the surface of the express package received by the customer will have the barcode face sheet for logistics information traceability. During the pick-up, the express delivery station staff shall log into the logistics information management platform through terminal device code scanning to record the information of "end of dispatch".

3) The Consumer Takes the Package and Delivers it to the Packaging Recycling Equipment

After the consumer takes the package, first inquire about the nearby recycling equipment, use the tools to remove the package as intact as possible in the equipment window, and then recycle and place it.

4) Out of Box Operations for Packaging Recycling Equipment

The recycling equipment has the function of automatically identifying the recycling space, and the operator can monitor the packaging recycling in the equipment in real time and facilitate timely out-of-box.

5 ) Traditional Recycling Department Recycling into Recirculation

After out of the box, third-party operators sell express delivery packages to traditional recycling institutions to obtain income, and express delivery packages enter ecommerce enterprises again through the processing process of circular reuse, forming a benign closed loop of the recycling system.

\subsection{System Courier Recycling Equipment Use}

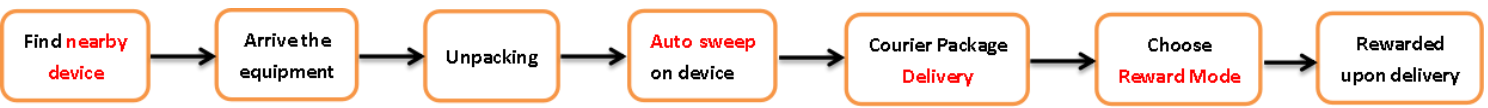

Figure 4 Operation process of recovery equipment of express package

As shown in Figure 4, with the support of Internet of Things technology and "Internet + " technology, the following process operations can be realized:

1) After taking the package, consumers can inquire (similar to the shared bicycle positioning inquiry service) the nearby recycling equipment on the mobile phone APP or applet (the client APP or applet supporting the operation and management of the express packaging recycling equipment system). The equipment is usually set next to the garbage sorting bucket (the investigation data shows about $95 \%$ recognition rate) and at the door of the neighborhood (similar to the position set up by the express delivery cabinet), which can facilitate customers to process the express packaging.

2) When the customer arrives at the equipment, the equipment window is specially equipped with the tools for unpacking, so as to help the customer to unpack and remove the goods as well as possible.

3 ) There is an operation instruction system (with hardware, Refer to the device hardware setting of the delivery cabinet) on the equipment, the customer selfservice code scanning is performed (bar code on the wrapped face sheet), the equipment delivery port is opened (2-3 ports of different sizes or types are divided according to different categories of recovery package )
, the equipment delivery port is opened, the customer delivers the package, and selects the incentive mode on the equipment operation interface (there are two incentive modes: one is that various points of the platform, such as shopping points, sunshine points, energy values, gold coins, etc., are used according to the use rules of each big online shopping platform; the other is cash red envelopes, random amount or the return amount of assisted packaging and recovery set by each big online shopping platform, etc.), and then the delivery port of the device is closed after clicking Confirm on the device operation interface.

4) The customer receives an incentive after delivering the courier package to the equipment for recycling.

\subsection{Operation mode of system}

The operation and management of recycled equipment adopts the mode of "government-led and third-party operation", that is, "the government provides equipment and the third party operates (it can be individuals, enterprises and various institutions including waste pickers, but it needs to pass the application and review and approval before the government issues the standard for review and approval)". Under this model, who operates and who opens the box. After the completion of out of box, the operator sells the express 
package to the waste recycling station in batches or directly to its upstream centralized treatment point, so that it can return to the end of the traditional recovery system and enter the resource recycling system.

\subsection{Stakeholder Chain of Systems}

In this operation model, the chain of interest relationships among governments, third parties, consumers, and e-commerce enterprises is shown in Figure 5.

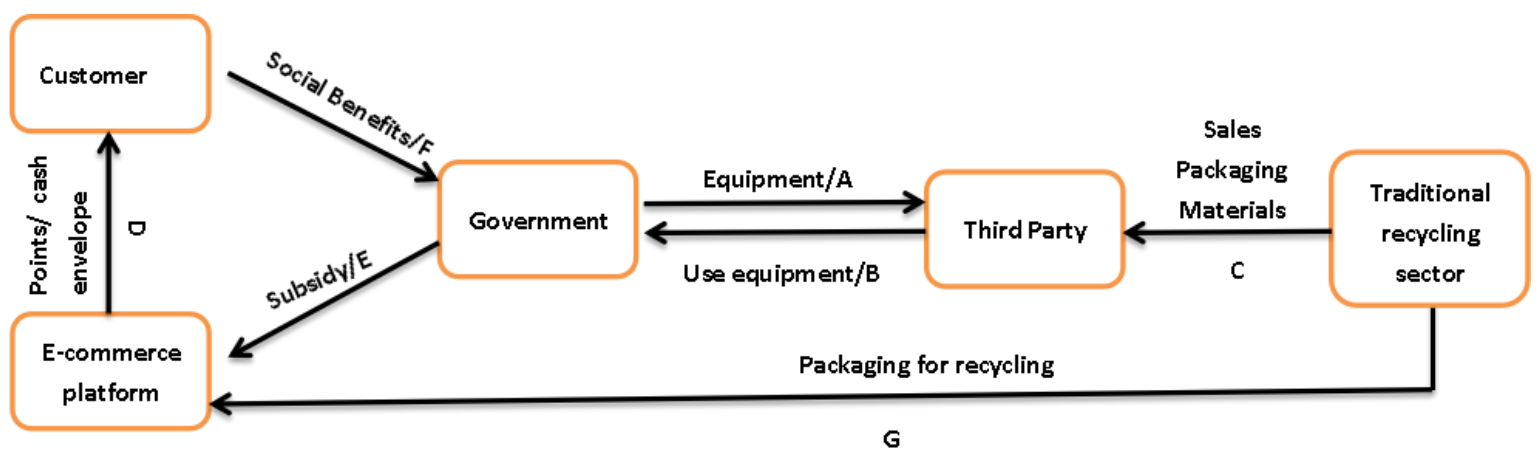

Figure 5 The Interest Chain of the Stakeholders of the Recovery System of the Recovery Equipment Based on the Courier Packaging

Among them: A- Total equipment investment cost of the government. B- Total equipment use cost paid by the third party to the government, such as rent, etc. C-Total income of packaging materials sold by the third party. DTotal score / cash red envelope reward returned by the ecommerce platform to consumers / customers. E-Total amount subsidized by the government to the e-commerce platform due to encouraging the development of green logistics. F- Social benefits brought about by consumers / customers actively assisting the development of green logistics, including the reduction of pollution, recycling and reuse of resources, relevant rewards given by the state, etc. G- Packaging for recycling and reuse purchased by e-commerce enterprises, saving the total cost.

In the interest chain, the operation rule is " $\mathrm{C}>\mathrm{B}>\mathrm{A}$, $\mathrm{C}>\mathrm{D}, \mathrm{F}>\mathrm{E} \geq \mathrm{D}, \mathrm{G}>0$ ":

1) For the government, the input cost is A and E, the income is $\mathrm{B}$ and $\mathrm{F}$, obviously $\mathrm{A}+\mathrm{E}<\mathrm{B}+\mathrm{F}$, is profitable.

2) For the third party, the cost of investment is $B$, the income is $\mathrm{A}$ and $\mathrm{C}$, obviously $\mathrm{B}<\mathrm{C}+\mathrm{A}$, it is profitable and can attract the third party to operate.

3 ) For customers, the input cost is zero or the delivery cost is very small, the income is D and the "social responsibility degree" similar to the current social credit (there is no, but there may be in the future), obviously 0 is less than $\mathrm{D}$, which is profitable.

4) For e-commerce platforms, the investment cost is $D$, the income is $E$ and $G$, and significantly $E+G \geq D$, which is profitable.

In summary, the whole system is a win-win state, and the joint action of all parties can realize government-led system linkage and realize the optimization and virtuous cycle of recovery system, so as to promote the recovery of express packaging materials and the sustainable development of green logistics.

\subsection{Expected results}

As shown in Figure 2, the recovery system designed based on the courier packaging equipment can achieve the following expected results:

1) Realizing the recycling of packaging express delivery materials solves the supply and demand contradiction of packaging materials to a certain extent, and can save some packaging material costs for ecommerce enterprises.

2) The new recycling system itself has the effect of guiding and motivating people to recover express packaging, which can actively improve people's action rate.

3 ) The new recycling system makes the recycling channels more specialized, so that the express packaging and garbage diversion. On the one hand, it can cultivate people's recycling awareness and habits, and on the other hand, it can also reduce the frequency of waste pickers turning garbage can, so as to reduce their health risks. At the same time, because waste pickers may also apply for becoming operators of recycling equipment, it can also help the optimal allocation of social human resources.

4 ) With the support of mature information technologies such as Internet of Things technology and "Internet +" technology, the logistics information of express products can be continued and directly converted into the "express packaging recovery information traceability" in the express packaging recovery system, helping the data statistics and analysis of recovery logistics.

5 ) Help the development of green logistics and 
recycling logistics. The new recycling system can help the awareness of environmental protection and garbage classification become popular, social environmental problems have been improved to a certain extent, the performance of the government environmental protection bureau has been improved, the pressure of the social security bureau has been reduced, more social personnel have been re-employed, and the society is more stable and beautiful.

\section{CONCLUSIONS}

Under the background of "environmental pollution + green logistics development demand" and the contradiction of express packaging supply less than demand, this paper explores a new recovery system - the recovery system based on express packaging recovery equipment. It takes the government as the leading factor, realizes the system linkage of multi-party cooperation, and can realize the win-win state. In addition to the mature Internet of Things technology and "Internet + " technology, it makes the implementation of this new recovery system feasible and operable.

At the same time, the recycling system will not only serve the recycling of express packaging in the future, but also serve the recycling of various recyclable resources. Although this paper provides only a conceptual model, lack of more specific empirical analysis and social practice, but to a certain extent, it can provide a strong reference for the relevant departments in the control of environmental pollution and the development of green logistics, in order to help the national scientific concept of development and "sustainable development" concept.

\section{CONSIDERATIONS}

First author: Jiagui Xie (March 1987), female, from Luzhou, Sichuan Province, master's degree, associate professor, mainly engaged in logistics engineering, transportation, teaching research.

Second author: Qin Chen (September 1999), female, from Chengdu, Sichuan Province, undergraduate, student, logistics engineering major.

Third author: Yu Yang (July 1999), female, Mianzhu, Sichuan Province, undergraduate, student, logistics engineering major.

\section{PROJECT FUND}

Sichuan Education Informatization Application and Development Research Center (Key Research Base for Joint Construction of Sichuan Education Department, Sichuan Social Science Association) 2020 Project (Project No.: JYXX20-017)

\section{REFERENCES}

[1] X. Wu, (2020) Analysis and Improvement of the Dilemma of Package Material Recovery by Express Delivery. Modern Marketing (Business Edition), (02):42.

[2] J. Yun, (2021) "Buy Buy Buy" Make the Carton in Short Supply! In the First Three Quarters, Japanese Corrugated Exports Increased by $90 \%$ [EB/OL]. https://new.qq.com/omn/20201110/20201110A0B X9V00.html

[3] China's Express Delivery Dusiness Volume Has Been the First in the World for Six Consecutive Years, and Packaging Green Governance Has Been Effective [J]. China Packaging, 2021, 41 (01): 20.

[4] R. Zheng, X. Sun, Y. Pan, (2020) Research and Construction of Courier Package Recycling System under Circulation Economy. Logistics Engineering and Management, 42(12): 1-4.

[5] N. Li, H. Fu, L. Liu, (2020) Present Situation and Existing Problems of Express Packaging in Ecommerce Environment. Printing Today, (12): 63 65.

[6] Eight Departments Accelerate the Green Transformation of Express Packaging. Green Package, 2021(01): 10-11. 